\title{
A new replacement name for the fossil spider Veterator Petrunkevitch, 1963 (Arthropoda: Arachnida: Araneae)
}

Un nuevo nombre sustitutivo para la araña fósil Veterator Petrunkevitch, 1963 (Arthropoda: Arachnida: Araneae)

\author{
Filippo CECCOLINI (1) \& Fabio CIANFERONI 우
}

\begin{abstract}
A junior homonym was detected in the genera of fossil Araneae and the following replacement name is proposed within the family Trochanteriidae: Neoveterator Ceccolini \& Cianferoni nom. nov. pro Veterator Petrunkevitch, 1963 nec Laws, 1944. Eight new combinations (comb. nov.) are proposed.
\end{abstract}

Resumen: Se ha detectado un homónimo más moderno entre los géneros fósiles de Araneae y se propone el siguiente nombre sustitutivo en la familia Trochanteriidae: Neoveterator Ceccolini \& Cianferoni nom. nov. pro Veterator Petrunkevitch, 1963 nec Laws, 1944. Se proponen ocho nuevas combinaciones (nov. comb.).
Received: 07 September 2021

Accepted: 28 October 2021

Published online: 19 November 2021

\section{Corresponding author:}

Fabio Cianferoni

fabio.cianferoni@cnr.it

Keywords:

Combinatio nova, Fossil Araneae Nomen novum, Replacement name

Palabras-clave:

Combinatio nova, Araneae fósiles Nomen novum, Nombre sustitutivo

\section{INTRODUCTION}

Arachnida is a very large group of arthropods including more than 112,000 living known species (Zhang, 2013), almost half of which represented by spiders (Araneae) (WSC, 2021). Although fossilization is not favored by their soft bodies (thus paleontological information of this group is relatively scarce) they likely represented a relevant proportion of past terrestrial biodiversity, as they do today (Dunlop \& Penny, 2012). Information about fossil arachnids was scattered until recent years, with the important exception of the wellknown, but outdated, contribution on spiders by Bonnet (1945, 1955, 1956, 1957, 1958, 1959, 1961); however, new complete lists were recently provided (Dunlop \& Penny, 2012; Dunlop et al., 2020). Dunlop et al. (2020) reported 2,154 fossil and subfossil recognized species of arachnids, with a significant increase of new descriptions in the last years (cf. Dunlop \& Penny, 2012; Dunlop et al., 2020). In the same catalog (Dunlop et al., 2020), 1,399 accepted species of extinct spiders are included. Among these, we noticed a junior homonym that, according to the rules of the International Code of Zoological Nomenclature (ICZN, 1999), needs to be replaced with new a name.

\section{REPLACEMENT NAME}

Petrunkevitch (1963, p. 24) proposed the new genus name Veterator for the new species $V$. extinctus Petrunkevitch, 1963 from Neogene Chiapas amber. Currently the name is accepted, including other seven species from Neogene Dominican amber (Dunlop et al., 2020).

However, the genus name created by Petrunkevitch is invalid, since it is a junior homonym of a genus name established by Laws (1944, p. 306) to accommodate the fossil species Brookesena quadricincta Marwick, 1931, a gastropod mollusc from New Zealand Miocene. Currently the name is accepted within the family Mathildidae (MolluscaBase, 2021).

Thus, Veterator Petrunkevitch, which does not have any available synonym, needs to be replaced (ICZN, 1999, Arts 60.1, 60.2): herein we propose Neoveterator Ceccolini \& Cianferoni nom. nov.

Etymology. The new name refers to the geological period of the fossil spider (Neogene), adding the prefix Neo- to the original name, which also remarks the nature of the new name (replacement name). Masculine gender.

(c) The Author(s) 2021. This is an open-access article distributed under the terms of the Creative Commons Attribution 4.0 International License (for details please see http://creativecommons.org/licenses/by/4.0/), which permits use, copy, adaptation, distribution, and reproduction in any medium or format, as long as you give appropriate credit to the original author(s) and the source. 


\section{SYSTEMATIC PALAEONTOLOGY}

Phylum ARTHROPODA von Siebold, 1848

Class ARACHNIDA Lamarck, 1801

Order ARANEAE Clerck, 1757

Family TROCHANTERIIDAE Karsch, 1879

Genus Neoveterator Ceccolini \& Cianferoni nom. nov. = Veterator Petrunkevitch, 1963 nec Laws, 1944

Included species. Neoveterator angustus (Wunderlich, 1988) comb. nov. = Veterator angustus Wunderlich, 1988

Neoveterator ascutum (Wunderlich, 1988) comb. nov. = Veterator ascutum Wunderlich, 1988

Neoveterator extinctus (Petrunkevitch, 1963) comb. nov. $=$ Veterator extinctus Petrunkevitch, 1963 (type species)

Neoveterator incompletus (Wunderlich, 1982) comb. nov. $=$ Veterator incompletus Wunderlich, 1982

Neoveterator longipes (Wunderlich, 1988) comb. nov. = Veterator longipes Wunderlich, 1988

Neoveterator loricatus (Wunderlich, 1988) comb. nov. = Veterator loricatus Wunderlich, 1988

Neoveterator porrectus (Wunderlich, 1988) comb. nov. = Veterator porrectus Wunderlich, 1988

Neoveterator viduus (Wunderlich, 1988) comb. nov. = Veterator viduus Wunderlich, 1988

Supplementary information. The article has no additional data.

Author contributions. The first author conceived the study. Both authors wrote the manuscript and equally contributed to the article. All authors approved the final version of the manuscript and agree to be held accountable for the content therein.

Competing Interest. We declare we have no competing interests.

Funding. We did not receive any funding for this study.

Author details. Filippo Ceccolini ${ }^{1}$ \& Fabio Cianferoni, ${ }^{1,2}$. 'Zoology, "La Specola", Natural History Museum, University of Florence, Via Romana 17, I-50125 Florence, Italy; ${ }^{2}$ Research Institute on Terrestrial Ecosystems, CNRNational Research Council of Italy, Via Madonna del Piano 10, I-50019 Sesto Fiorentino (Florence), Italy; ceccolinif@ virgilio.it; fabio.cianferoni@cnr.it

Acknowledgments. We are grateful to the Editor in chief Carlos Martínez-Pérez (Universidad de Valencia, Spain) for his kind help during the review process. We are also pleased to thank Enrique Peñalver Moyá (Instituto Geológico y Minero de España, Valencia, Spain) and an anonymous reviewer for their valuable revisions which improved the manuscript.

\section{REFERENCES}

Bonnet, P. (1945). Bibliographia Araneorum. Analyse méthodique de toute la littérature aranéologique jusqu'en. Tome I. Douladoure.

Bonnet, P. (1955). Bibliographia Araneorum. Analyse méthodique de toute la littérature aranéologique jusqu'en. Tome II. Systématique des araignées (Étude par ordre alphabétique). $1^{r e}$ partie: $A-B$. Douladoure.

Bonnet, P. (1956). Bibliographia Araneorum. Analyse méthodique de toute la littérature aranéologique jusqu'en. Tome II. Systématique des araignées (Étude par ordre alphabétique). $2^{\text {re }}$ partie: C-F. Douladoure.

Bonnet, P. (1957). Bibliographia Araneorum. Analyse méthodique de toute la littérature aranéologique jusqu'en. Tome II. Systématique des araignées (Étude par ordre alphabétique). $3^{\text {re }}$ partie: G-M. Douladoure.

Bonnet, P. (1958). Bibliographia Araneorum. Analyse méthodique de toute la littérature aranéologique jusqu'en. Tome II. Systématique des araignées (Étude par ordre alphabétique). $4^{\text {re }}$ partie: N-S. Douladoure.

Bonnet, P. (1959). Bibliographia Araneorum. Analyse méthodique de toute la littérature aranéologique jusqu'en. Tome II. Systématique des araignées (Étude par ordre alphabétique). $5^{\text {re }}$ partie: T-Z. Douladoure.

Bonnet, P. (1961). Bibliographia Araneorum. Analyse méthodique de toute la littérature aranéologique jusqu'en. Tome III. Index alphabétiques, résultats - conclusions, considérations diverses. Douladoure.

Clerck, C. (1757). Svenska Spindlar uti sina hufvud-slågter indelte samt under några och sextio särskildte arter beskrefne och med illuminerade figurer uplyste / Aranei Svecici, descriptionibus et figuris æneis illustrati, ad genera subalterna redacti, speciebus ultra $L X$ determinati. Laurentius Salvius.

Dunlop, J. A., \& Penny, D. (2012). Fossil Arachnids. Siri Scientific Press.

Dunlop, J. A., Penny, D., \& Jekel, D. (2020). A summary list of fossil spiders and their relatives. In World Spider Catalog. Natural History Museum Bern, online at http://wsc.nmbe. ch, version 20.5, accessed on 25 August 2021.

ICZN, International Commission on Zoological Nomenclature (1999). International Code of Zoological Nomenclature. 4th Edition. The International Trust for Zoological Nomenclature.

Karsch, F. (1879). Arachnologische Beitrage. Zeitschrift für die Gesammten Naturwissenschaften, 52, 534-562.

Lamarck, J. B. (1801). Système des animaux sans vertèbres, ou tableau général des classes, des ordres et des genres de ces animaux; Présentant leurs caractères essentiels et leur distribution, d'apres la considération de leurs rapports naturels et de leur organisation, et suivant l'arrangement établi dans les galeries du Muséum d'Histoire Naturelle, parmi leurs dépouilles conservées; Précédé du discours d'ouverture du Cours de Zoologie, donné dans le Muséum National d'Histoire Naturelle l'an 8 de la République. Published by the author and Deterville.

Laws, C. R. (1944). The molluscan faunule at Pakaurangi Point, Kaipara, No. 3. Transactions and Proceedings of the Royal Society of New Zealand, 76, 537-541. 
Marwick, J. (1931). The Tertiary Mollusca of the Gisborne District. New Zealand Geological Survey Paleontological Bulletin, 13, 1-177.

MolluscaBase (Eds.) (2021). MolluscaBase. Accessed at http://www.molluscabase.org [last accessed 24 August 2021]. doi: $10.14284 / 448$

Petrunkevitch, A. (1963). Chiapas amber spiders. University of California Publications in Entomology, 31, 1-40.

von Siebold, C. T. (1848). Lehrbuch der vergleichenden Anatomie der Wirbellosen Thiere. Erster Theil. In C. T. von Siebold, \& H. Stannius (Eds.), Lehrbuch der vergleichenden Anatomie (pp.1-679). Verlag von Veit \& Comp.

WSC, World Spider Catalog (2021). World Spider Catalog. Version 22.5. Bern: Natural History Museum, online at https://wsc.nmbe.ch [Last accessed 24 August 2021]. doi: $10.24436 / 2$
Wunderlich, J. (1982). Die häufigsten Spinnen (Araneae) des Dominikanischen Bernsteins. Neue Entomologische Nachrichten, 1, 26-45.

Wunderlich, J. (1988). Die fossilen Spinnen im dominikanischen Bernstein. Beiträge zur Araneologie, 2, 1-378.

Wunderlich, J. (2004). Fossil jumping spiders (Araneae: Salticidae) in Baltic and Dominican amber, with remarks on Salticidae subfamilies. In J. Wunderlich (Ed.), Fossil spiders in amber and copal. Beiträge zur Araneologie, 3, 1761-1819.

Zhang, Z.-Q. (Ed.) (2013). Animal Biodiversity: An Outline of Higher-level Classification and Survey of Taxonomic Richness (Addenda 2013). Zootaxa, 3703(1), 1-82. doi: 10.11646/zootaxa.3703.1.6 
